Proyecciones Journal of Mathematics

Vol. 33, No 4, pp. 405-413, December 2014.

Universidad Católica del Norte

Antofagasta - Chile

\title{
On linear maps that preserve $G$-partial-isometries in Hilbert space
}

\author{
Abdellatif Chahbi \\ University of Ibn Tofail, Morocco \\ and \\ Samir Kabbaj \\ University of Ibn Tofail, Morocco \\ Received: Abril 2014. Accepted : July 2014
}

\begin{abstract}
Let $\mathcal{H}$ be a complex Hilbert space and $\mathcal{B}(\mathcal{H})$ the algebra of all bounded linear operators on $\mathcal{H}$. We give the concrete forms of surjective continue unital linear maps from $\mathcal{B}(\mathcal{H})$ onto itself that preserves G-partial-isometric operators.
\end{abstract}

Key words and phrases : Linear preserver, Jordan homomorphisms, Operators on spaces with an indefinite metric, partialisometric operators.

2010 Mathematics Subject Classifications : Primary 15A86; 15A04; 47B50 Secondary 47L30; 16W20. 


\section{Introduction}

Linear preserver problems is an active research area in Matrix, operator theory and Banach algebras. It has attracted the attention of many mathematicians in the last few decades ([3]-[9], [11, 13], [14] - [19] ). By a linear preserver we mean a linear map of an algebra $\mathcal{A}$ into itself which, roughly speaking, preserves certain properties of some elements on $\mathcal{A}$.

Linear preserver problems concern the characterization of such maps. Automorphisms and anti-automorphisms certainly preserve various properties of the elements. Therefore, it is not surprising that these two types of maps often appear in the conclusions of the results. In this paper, we shall concentrate on the case when $\mathcal{A}=\mathcal{B}(\mathcal{H})$, the algebra of all bounded linear operators on a complex Hilbert space $\mathcal{H}$. We should point out that a great deal of work has been devoted to the case when $\mathcal{H}$ is finite dimensional,that is, the case when $\mathcal{A}$ is a matrix algebra (see survey articles $[15,11,12]$ ), and that the first papers concerning this case date back to the previous century [9].

The aim of this paper is to characterize surjective continuous linear maps

$$
\phi: \mathcal{B}(\mathcal{H}) \rightarrow \mathcal{B}(\mathcal{H})
$$

preserving $G$-partial-isometries.

\section{Preliminaries}

Let $(\mathcal{H},[]$,$) be an indefinite inner product space (\mathrm{cf}[1],[2],[20])$. It is well known that $(\mathcal{H},[]$,$) is a complete indefinite inner product if and only if \mathcal{H}$ is a Hilbert space with some inner product \langle\rangle and there exists an invertible self-adjoint operator $G \in \mathcal{B}(\mathcal{H})$ such that

$$
[x, y]=[x, y]_{G}=\langle G x, y\rangle
$$

for all $x, y \in \mathcal{H}$, and the set of all bounded linear operators on $\mathrm{H}$ with respect to the indefinite inner product [,] is the same as $\mathcal{B}(\mathcal{H},\langle\rangle$,$) . Thus,$ we may always assume that $\mathcal{H}$ is a Hilbert space with inner product $\langle$,$\rangle .$ For an invertible self-adjoint operator $G$ and $A \in \mathcal{B}(\mathcal{H})$, denote $A^{\sharp}$ the indefinite adjoint of $A$ with respect to $G$, i.e., the $G$-adjoint of $A$, which is determined by

$$
\left[x, A^{\sharp} y\right]_{G}=[A x, y]_{G}
$$

for all $x, y \in \mathcal{H}$. Clearly, $A^{\sharp}=G^{-1} A^{*} G$, where $A^{*}$ is the adjoint of $A$ with respect to the inner product \langle\rangle . 
We continue by definition and theorem of $G$-partial-isometric operator (cf $[8])$.

Definition 2.1. An operator $V$ on $\mathcal{B}(\mathcal{H})$ is called $G$-partial-isometry if and only if

$$
V V^{\sharp} V=V .
$$

Theorem 2.2. Let $V \in \mathcal{B}(\mathcal{H})$. The following assertions are equivalent:

1. $V$ is a $G$-partial isometry.

2. $V^{\sharp}$ is a $G$-partial isometry.

3. $V V^{\sharp}$ is a $G$-projection.

4. $V^{\sharp} V$ is a $G$-projection.

5. $V^{\sharp} V^{\sharp} V=V^{\sharp}$.

6. $V V^{\sharp} V=V$.

A linear map $\phi$ from algebra $\mathcal{A}$ into an algebra $\mathcal{B}$ is called a Jordan homomorphism if $\phi\left(x^{2}\right)=\phi(x)^{2}$ for every $x \in \mathcal{A}$. A well known result of Herstein ([10], Theorem 3.1) shows that a Jordan homomorphism on prime algebra is either a homomorphism or an anti-homomorphism.

\section{Main Result}

First we prove the following lemmas which are useful for the proof of the main theorems.

Lemma 3.1. For all $G$-self-adjoint $S \in \mathcal{B}(\mathcal{H}), \exp (i t S)$ is $G$-unitary operator for every $t \in \mathbf{R}$.

Proof. See (Lemma 3.1, [7] ).

Lemma 3.2. Let $\mathcal{H}$ be a separable complex Hilbert space. If $\phi: \mathcal{B}(\mathcal{H}) \rightarrow$ $\mathcal{B}(\mathcal{H})$ is a continuous and surjective homomorphism or anti-homomorphism then $\phi$ is injective. 
Proof. See (Lemma 3.2, [7] ).

Theorem 3.3. Let $\mathcal{H}$ be a separable complex Hilbert space and let $\phi$ : $\mathcal{B}(\mathcal{H}) \rightarrow \mathcal{B}(\mathcal{H})$ be a unital linear continuous surjective map. If $\phi$ preserves $G$-partial-isometric operators in one direction, then there exist $\lambda= \pm 1$ and invertible operator $U \in \mathcal{B}(\mathcal{H})$ satisfying $U U^{\sharp}=U^{\sharp} U=\lambda I$ such that

$$
\phi(A)=\lambda U A U^{\sharp},
$$

or

$$
\phi(A)=\lambda U A^{t} U^{\sharp}
$$

for all $A \in \mathcal{B}(\mathcal{H})$, where $A^{t}$ is the transpose of $A$, with respect to an arbitrary but fixed orthonormal base of $\mathcal{H}$.

Proof. $\quad$ Pick a $G$-self-adjoint operator $S$ in $\mathcal{B}(\mathcal{H})$. By Lemma 3.1 $\exp (i t S)$ is a $G$-unitary operator for every $t \in \mathbf{R}$, so $\exp (i t S)$ is $G$-partialisometry. Therefore,

$$
\begin{aligned}
& \phi(\exp (i t S))=\phi(\exp (i t S)) \phi(\exp (i t S))^{\sharp} \phi(\exp (i t S)) \\
= & \phi\left(I+i t S+\frac{(i t)^{2}}{2 !} S^{2}+\ldots\right) \phi\left(I+i t S+\frac{(i t)^{2}}{2 !} S^{2}+\ldots\right)^{\sharp} \\
& \phi\left(I+i t S+\frac{(i t)^{2}}{2 !} S^{2}+\ldots\right) \\
= & I+i t\left(2 \phi(S)-\phi(S)^{\sharp}\right)+t^{2}\left(\phi(S)^{\sharp} \phi(S)+\phi(S) \phi(S)^{\sharp}\right. \\
- & \left.\frac{1}{2} \phi\left(S^{2}\right)^{\sharp}-\phi\left(S^{2}\right)-\phi(S)^{2}\right) \ldots
\end{aligned}
$$

Hence

$$
\begin{gathered}
I+i t \phi(S)-\frac{t^{2}}{2} \phi\left(S^{2}\right) \ldots= \\
I+i t\left(2 \phi(S)-\phi(S)^{\sharp}\right)+t^{2}\left(\phi(S)^{\sharp} \phi(S)+\phi(S) \phi(S)^{\sharp}\right. \\
\left.-\frac{1}{2} \phi\left(S^{2}\right)^{\sharp}-\phi\left(S^{2}\right)-\phi(S)^{2}\right) \ldots
\end{gathered}
$$

It follows that

$$
\phi(S)=\phi(S)^{\sharp}
$$

and

$$
\phi(S)^{\sharp} \phi(S)+\phi(S) \phi(S)^{\sharp}-\frac{1}{2} \phi\left(S^{2}\right)^{\sharp}-\frac{1}{2} \phi\left(S^{2}\right)-\phi(S)^{2}=0
$$


for every $G$-self-adjoint operator $S$.

For any $A \in \mathcal{B}(\mathcal{H})$ we can write $A=S+i T$ with $S, T$ are $G$-self-adjoint operators. We find from (3.1) and the linearity of $\phi$ that

$$
\begin{aligned}
\phi\left(A^{\sharp}\right) & =\phi(S-i T) \\
& =\phi(S)-i \phi(T) \\
& =\phi(S)^{\sharp}-i \phi(T)^{\sharp} \\
& =\phi(S+i T)^{\sharp} \\
& =\phi(A)^{\sharp} .
\end{aligned}
$$

It follows that $\phi\left(A^{\sharp}\right)=\phi(A)^{\sharp}$ for all $A \in \mathcal{B}(\mathcal{H})$. Now we will prove that $\phi$ is a Jordan homomorphism. By (3.1) and (3.2), it is easy to deduce that

$$
\phi\left(S^{2}\right)=\phi(S)^{2}
$$

for every $G$-self-adjoint operator $S$. Since every operator $A \in B(\mathcal{H})$ is written in the form $A=S+i T$ with $S, T$ are $G$-self-adjoint, $S+T$ is $G$-selfadjoint operator, hence

$$
\phi\left((S+T)^{2}\right)=(\phi(S)+\phi(T))^{2},
$$

and so

$$
\phi(S T+T S)=\phi(S) \phi(T)+\phi(T) \phi(S)
$$

consequently

$$
\begin{aligned}
\phi\left(A^{2}\right) & =\phi\left((S+i T)^{2}\right) \\
& =\phi\left(S^{2}-T^{2}+i(S T+T S)\right) \\
& =\phi\left(S^{2}\right)-\phi\left(T^{2}\right)+i \phi(S T+T S) \\
& =\phi(S)^{2}-\phi(T)^{2}+i \phi(S) \phi(T)+\phi(T) \phi(S) \\
& =\phi(S+i T)^{2} \\
& =\phi(A)^{2}
\end{aligned}
$$

It follows that $\phi\left(A^{2}\right)=\phi(A)^{2}$, for all operator $A$ in $\mathcal{B}(\mathcal{H})$, which implies that $\phi$ is a Jordan homomorphism. But it is known that every Jordan homomorphism in prime algebra is a homomorphism or an antihomomorphism. Since $\mathcal{B}(\mathcal{H})$ is a prime algebra, then $\phi$ is a homomorphism 
or an anti-homomorphism. By Lemma $3.2 \phi$ is injective, so $\phi$ is an automorphism or an anti-automorphism. Note that an automorphism or an anti-automorphism in $\mathcal{B}(\mathcal{H})$ is written in the form

$$
\phi(A)=U A U^{-1}
$$

for all $A$ in $\mathcal{B}(\mathcal{H})$, or

$$
\phi(A)=U A^{t} U^{-1}
$$

for all $A$ in $\mathcal{B}(\mathcal{H})$, where $U$ is an invertible operator. We only consider the first form of $\phi$, the proof of the second form is similar to the first. Moreover, $\phi$ is a $G$-self-adjoint i.e. $\phi\left(A^{\sharp}\right)=\phi(A)^{\sharp}$, so

$$
U G A^{*} G^{-1} U^{-1}=G\left(U^{-1}\right)^{*} A^{*} U^{*} G^{-1}
$$

for all $A$ in $\mathcal{B}(\mathcal{H})$. We can get that

$$
U^{*} G^{-1} U G A^{*}=A^{*} U^{*} G^{-1} U G .
$$

for all $A$ in $\mathcal{B}(\mathcal{H})$. Since the center of $\mathcal{B}(\mathcal{H})$ is the set of scalar operators, $U^{*} G U G^{-1}=\lambda I$. Then $U^{\sharp} U=\lambda I$, since $U$ is an invertible operator, then also $U U^{\sharp}=\lambda I$. Since $U^{\sharp} U$ is a $G$-self-adjoint operator and $U$ is an invertible operator so $\lambda$ is a nonzero real scalar. In order to complete, the proof can be reduced to $\lambda= \pm 1$, which will complete the proof.

When the Hilbert spaces is not necessarily separable, we have the following result.

Theorem 3.4. Let $\mathcal{H}$ be a complex Hilbert space and let $\phi: \mathcal{B}(\mathcal{H}) \rightarrow \mathcal{B}(\mathcal{H})$ be a unital linear continuous surjective map. If $\phi$ preserves $G$-partialisometries in both directions, then there exist $\lambda= \pm 1$ and invertible operator $U \in \mathcal{B}(\mathcal{H})$ satisfying $U U^{\sharp}=U^{\sharp} U=\lambda I$ such that

$$
\phi(A)=\lambda U A U^{\sharp},
$$

or

$$
\phi(A)=\lambda U A^{t} U^{\sharp}
$$

for all $A \in \mathcal{B}(\mathcal{H})$, where $A^{t}$ is the transpose of $A$, with respect to an arbitrary but fixed orthonormal base of $\mathcal{H}$.

First we prove the following result which is useful in the proof of this theorem.

Lemma 3.5. $\phi$ is injective. 
Proof. Let $T \in \mathcal{B}(\mathcal{H})$ such that $\phi(T)=0$, then $\phi(\lambda T)=0$ for all $\lambda \in \mathbf{C}$. Since $\phi$ preserve $G$-partial-isometries, so $\lambda T$ is a $G$-partial-isometry for all $\lambda \in \mathbf{C}$. It follow that

$$
\lambda T \bar{\lambda} T^{\sharp} \lambda T=\lambda T,
$$

this implies that

$$
|\lambda|^{2} T T^{\sharp} T=T .
$$

Taking successively $\lambda=1$ and $\lambda=2$, we get

$$
T T^{\sharp} T=T
$$

and

$$
4 T T^{\sharp} T=T .
$$

A simple calculus gives that $T=0$, hence the proof is complete. $\square$ We will prove the theorem 3.4.

Proof. By Lemma 3.5, $\phi$ is injective so $\phi$ is a continuous unital bijective linear map that preserves the $G$-partial-isometries in both directions. Now as in the proof of Theorem 3.3 we obtain the form of $\phi$.

\section{References}

[1] T. Ya. Azizov, I. S. Iokhvidov: Linear Operators in Spaces with an Indefinite Metric. John Wiley \& Sons, Chichester, New York, (1989).

[2] J. Bognar, Indefinite Inner Product Spaces. Springer, Berlin, (1974).

[3] M. Brešar and C. R. Miers, Commutativity preserving mappings of von Neumann algebras, Canad. J. Math. 45, pp. 695-708, (1993).

[4] M. Brešar and P. Šemrl, Mappings which preserve idempotents, local automorphisms, and local derivations, Canad. J. Math. 45, pp. 483496, (1993).

[5] M. Brešar and P. Šemrl, Linear maps preserving the spectral radius, J. Funct. Anal. 142, pp. 360-368, (1996). 
[6] M.Brešar and P.Šemrl, Linear preservers on B(X), Banach Cent. Publ. 38, pp. 49-58, (1997).

[7] A. Chahbi \& S.Kabbaj, Linear maps preserving G-unitary Operators in Hilbert space, Arab Journal of Mathematical Sciences, (2014). (in press)

[8] M. A. Dritschel \& J. Rovnyak, Operators on indefinite inner product spaces, Lectures on operator theory and its applications 3, pp. 141-232, (1996).

[9] G. Frobenius, $\ddot{U}$ ber die Darstellung der endlichen Gruppen durch lineare Substitutionen, Sitzungsber. Deutsch. Akad. Wiss. Berlin, pp. 994-1015, (1897).

[10] I. N. Herstein, Topics in ring theory, University of Chicago Press, Chicago, (1969).

[11] C.-K. Li and N. K. Tsing, Linear preserver problems: A brief introduction and some special techniques, Linear algebra and its applications 162, pp. 217-235, (1992).

[12] M. Marcus, Linear operations on matrices, Amer. Math. Monthly 69, pp. 837-847, (1962).

[13] M. Mbekhta, Linear maps preserving the generalized spectrum, Extracta Mathematicae 22, pp. 45-54, (2007).

[14] M. Omladič and P.Šemrl, Linear mappings that preserve potent operators, Proc. Amer. Math. Soc. 123, pp. 1069-1074, (1995).

[15] S. Pierce, M. H. Lim, R. Loewy, C. K. Li, N. K. Tsing, B. McDonald, \& L.Beasley, A survey of linear preserver problems, Linear and Multilinear Algebra 33, pp. 1-129, (1992).

[16] M. Rais, The unitary group preserving maps (the infinite-dimensional case), Linear and Multilinear Alg. 20, 4, pp. 337-345, (1987).

[17] B. Russo and H. A. Dye, A note on unitary operators in $C^{*}$-algebras, Duke Math. J. 33, pp. 413-416, (1966).

[18] P. Šmrl, Two characterizations of automorphisms on $B(X)$, Studia Math. 105, pp. 143-149, (1993). 
[19] W.Watkins, Linear maps that preserve commuting pairs of matrices, Linear Algebra Appl. 14, pp. 29-35, (1976).

[20] D.X. Xia, S.Z. Yan, Spectrum Theory of Linear operators II: Operator Theory on Indefinite Inner Product Spaces, Science Press, Beijing, (1987).

\author{
Abdellatif Chahbi \\ Department of Mathematics, \\ Faculty of Sciences, \\ University of Ibn Tofail, \\ Kenitra, \\ Morocco \\ e-mail : ab $1980 @ l i v e . f r$ \\ and \\ Samir Kabbaj \\ Department of Mathematics, \\ Faculty of Sciences, \\ University of Ibn Tofail, \\ Kenitra, \\ Morocco \\ e-mail : samkabbaj@yahoo.fr
}

